\title{
What can tracer observations in the continental boundary layer tell us about surface-atmosphere fluxes?
}

\author{
C. Gerbig ${ }^{1}$, J. C. Lin ${ }^{2,}$, J. W. Munger ${ }^{2}$, and S. C. Wofsy ${ }^{2}$ \\ ${ }^{1}$ Max-Planck-Institute for Biogeochemistry, P.O. Box 1001 64, 07701 Jena, Germany \\ ${ }^{2}$ Dept. of Earth and Planetary Sciences and Division of Engineering and Applied Sciences, Harvard University, Cambridge, \\ MA, USA \\ *now at: Department of Atmospheric Science, Colorado State University, 1371 Campus Delivery, Fort Collins, CO \\ 80523-1371, USA
}

Received: 19 July 2005 - Published in Atmos. Chem. Phys. Discuss.: 27 September 2005

Revised: 10 January 2006 - Accepted: 17 January 2006 - Published: 22 February 2006

\begin{abstract}
We analyze the potential for inferring spatially resolved surface fluxes from atmospheric tracer observations within the mixed layer, such as from monitoring towers, using a receptor oriented transport model (Stochastic TimeInverted Lagrangian Transport model - STILT) coupled to a simple biosphere in which $\mathrm{CO}_{2}$ fluxes are represented as functional responses to environmental drivers (radiation and temperature). Transport and biospheric fluxes are coupled on a dynamic grid using a polar projection with high horizontal resolution $(\sim 20 \mathrm{~km})$ in near field, and low resolution far away (as coarse as $2000 \mathrm{~km}$ ), reducing the number of surface pixels without significant loss of information. To test the system, and to evaluate the errors associated with the retrieval of fluxes from atmospheric observations, a pseudo data experiment was performed. A large number of realizations of measurements (pseudo data) and a priori fluxes were generated, and for each case spatially resolved fluxes were retrieved. Results indicate strong potential for high resolution retrievals based on a network of tall towers, subject to the requirement of correctly specifying the a priori uncertainty covariance, especially the off diagonal elements that control spatial correlations. False assumptions about the degree to which the uncertainties in the a priori fluxes are spatially correlated may lead to a strong underestimation of uncertainties in the retrieved fluxes, or, equivalently, to biased retrievals. The framework presented here, however, allows a conservative choice of the off diagonal elements that avoids biasing the retrievals.
\end{abstract}

Correspondence to: C. Gerbig

(cgerbig@bgc-jena.mpg.de)

\section{Introduction}

Climate predictions are currently hampered by the inability to characterize feedback between a changing climate and sources and sinks of greenhouse gases such as $\mathrm{CO}_{2}$ and methane (Friedlingstein et al., 2003). A prerequisite for development of a predictive capability is the understanding of current trace gas budgets. At hemispheric scales, a major source of information has been data from the $\mathrm{CO}_{2}$ observing network (e.g. GLOBALVIEW- $\mathrm{CO}_{2}, 2002$ ), which are used by atmospheric transport models in inversions to derive flux estimates. However, at the smaller scales of continents, countries or ecosystems, at which climate anomalies (droughts, anomalies in rainfall, temperature, etc.) as well as human intervention (land use change) influence biosphere-atmosphere exchange, large uncertainties remain (IPCC, 2001; Schimel et al., 2001). Regional scale budgets have recently become a research focus (Wofsy and Harriss, 2002); they are a requirement for any carbon trading, such as might be implemented under international agreements like the Kyoto protocol.

Continental scale inversions based on global observational networks provide only a weak constraint on fluxes, due to the remoteness of such observations from the land (Gloor et al., 2000). In principle, information about regional scale biosphere-atmosphere exchange is contained in mixing ratios of $\mathrm{CO}_{2}$ over the continent, in proximity of biospheric activity. Gerbig et al. (2003a) (in the following referred to as G03a) demonstrated large biospheric signals in atmospheric profiles of $\mathrm{CO}_{2}$ over the North American continent, with a significant spatial variability that closely reflects spatial variability in surface fluxes (Gerbig et al., 2003b, in the following referred to as G03b). 

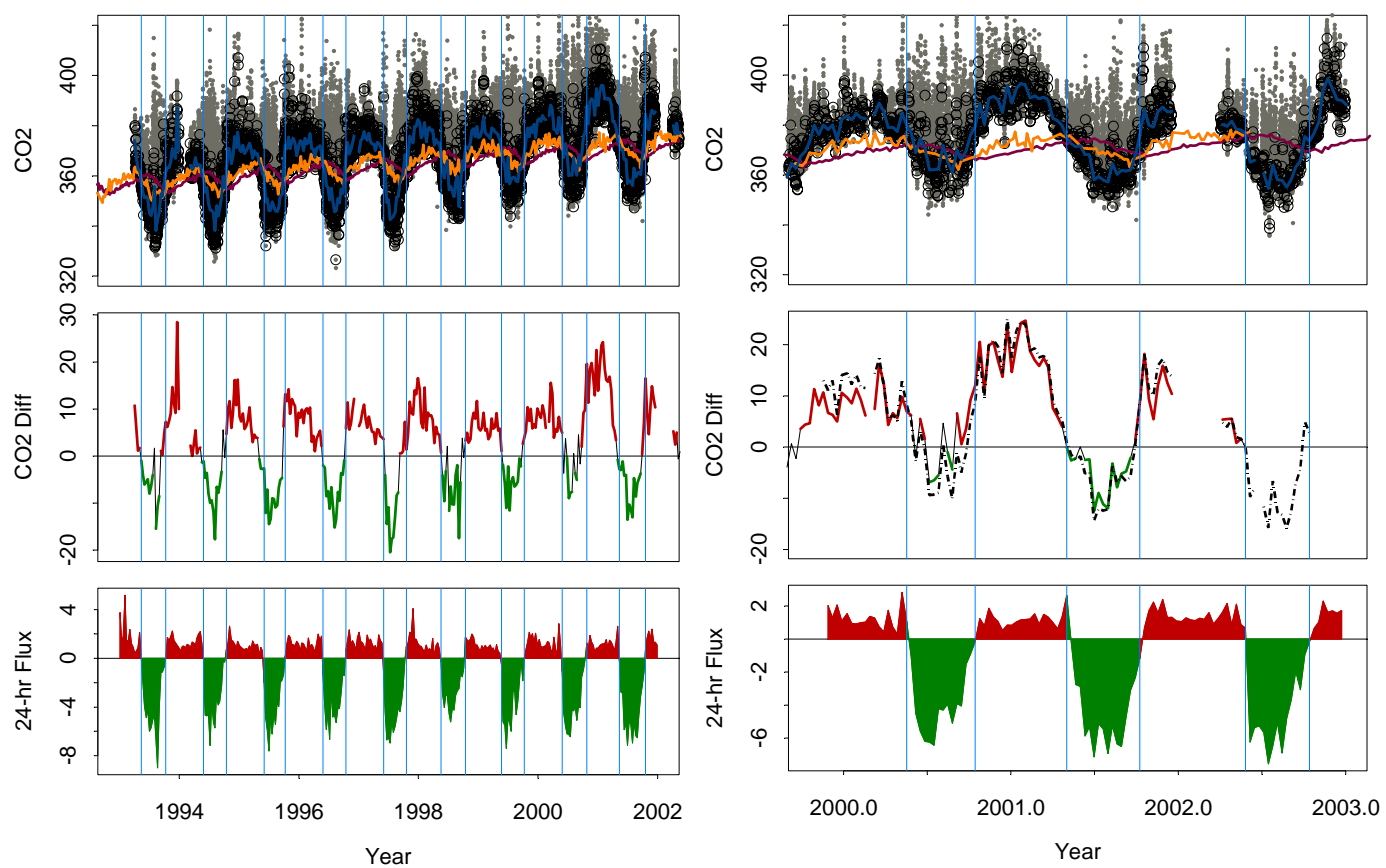

Fig. 1. Concentrations of $\mathrm{CO}_{2}$ at the Harvard Forest tower compared to remote station and aircraft data. Upper left: hourly $\mathrm{CO}_{2}(\mathrm{ppm})$ at $30 \mathrm{~m}$ [grey dots], midday values [black circles], 10-day medians of the midday data [blue line], and 10-day medians from Bermuda East [orange line] and Mauna Loa [red line]. Middle left: difference ( $\mathrm{ppm})$ between midday Harvard Forest concentrations and Bermuda E, colorcoded by the sign of the $\mathrm{CO}_{2}$ flux (green=uptake, red=emission). Lower left: 10-day median of the daily mean $\mathrm{CO}_{2}$ flux at Harvard Forest $\left(\mu\right.$ mole $\mathrm{m}^{-2} \mathrm{~s}^{-1}$ ). Right collumn: same as left, expanded time axis, with addition of data from $3 \mathrm{~km}$ altitude from aircraft sampling over Harvard Forest [black line dotted]. (Bermuda, Mauna Loa, and aircraft data from T. Conway, NOAA Climate Monitoring and Diagnostics Laboratory, 2004).

Long-term observations of $\mathrm{CO}_{2}$ in the continental boundary layer with sufficient accuracy are available from tall towers (Bakwin et al., 1995) as well as from a number of $\mathrm{CO}_{2}$ eddy flux towers (Fig. 1), and the spatial density of this network of sites is increasing (Wofsy and Harriss, 2002). These data are closely related to regional fluxes of $\mathrm{CO}_{2}$, especially when they can be compared to observations from above the planetary boundary layer (PBL) and/or remote stations at similar latitudes (Fig. 1, compare middle and lower panels). Unfortunately, the large spatial as well as temporal variability of the fluxes limits the usefulness of these data in current models used for inversions, and interpretation of the observations requires quantitative understanding of transport processes affecting the PBL. Most inversions so far have used a representation of surface fluxes where surface grid elements are aggregated to large regions (typically 10-20 globally) (Gurney and al., 2002), and PBL processes are not accurately represented; these inversions are subject to additional representation errors (the so-called "aggregation error"; Kaminski et al., 2001). G03b showed that changes in $\mathrm{CO}_{2}$ concentrations over the continent are directly related to spatial variations of surface fluxes in the near field of the measurement locations. To quantitatively extract the information, aggregation to coarse spatial scales should be avoided. Otherwise the aggregation error has to be accounted for in the inversion, and (as G03b argued) this might involve not just a random error but also a bias term.

Bayesian inversion methods require as inputs an a priori estimate of the state (in this case the surface fluxes) and an associated error covariance matrix that characterizes the uncertainties in the estimate (Rodgers, 2000). When retrieving flux information at high spatial resolution, special attention has to be given to this covariance matrix, most importantly the off-diagonal elements. This can be illustrated with two extremes: a) Assuming no correlation between different locations (i.e. zero off-diagonal elements) implies very small a priori uncertainties on large scales, since uncorrelated errors are reduced by factor $1 / \mathrm{N}^{-1 / 2}$, with $\mathrm{N}$ as the number of gridcells aggregated. For a large number of independent gridcells (large degrees of freedom) the atmospheric inversion will be less successful in constraining fluxes; in fact the problem becomes underdetermined (Peylin et al., 2001); b) assuming perfect correlation between different locations means there is only one degree of freedom (a bias) to be constrained for each large scale aggregate by the inversion. Large scale aggregation (such as used in the TransCom studies (Gurney and al., 2002) is equivalent to the assumption of perfect uncertainty covariance within each aggregate. But, as illustrated 
in Fig. 1, data from continental sites are sensitive to regional fluxes, and this approach would render these data useless in the inversion. A realistic covariance of the prior uncertainty will fall between these two extremes.

A direct way to derive the uncertainty covariance would be to compare the prior flux estimate to measurements at various locations, and to investigate the spatial and temporal correlation of the residuals (differences between measurement and flux estimate). However, this requires a dense network of flux measurement sites in a spatial arrangement that allows for spatial statistics, e.g. multiple replica within similar ecosystems at various distances. Lacking observational constraints for the prior uncertainty covariance, one has to make assumptions about the spatial correlations, e.g. in the form of a correlation length scale, for example, adopting the spatial statistics of differences between different biospheric models (Rödenbeck et al., 2003). Alternatively, correlation length scales have been estimated by optimization of the modeldata system using a geostatistical approach (Michalak et al., 2004). Both approaches give only a surrogate for true uncertainties and their spatial covariances. In a recent study on regional scale inversions (Peylin et al., 2005) the assumed correlation length scale was varied between 500 and $2000 \mathrm{~km}$, yielding significantly different flux distributions.

To investigate the inference of $\mathrm{CO}_{2}$ flux from measurements of $\mathrm{CO}_{2}$ over the continent (e.g. the data in Fig. 1), and especially the dependence on the assumed error characteristic, we set up the ROAM (receptor-oriented atmospheric model) framework described in G03b to retrieve fluxes at high spatial resolution. ROAM couples a high resolution transport model (the Stochastic Time-Inverted Lagrangian Transport model, STILT, Lin et al., 2003) to a simple biosphere flux model intended to capture the temporal and spatial variance over the continent. The flux model used here, denoted the "Greatly Simplified Biosphere" (GSB), parameterizes net ecosystem exchange as a response to radiation and temperature, similar to G03b, except that here we solve for temperature and light sensitivities directly rather than for scaling factors of respiration fluxes and uptake. A further, but more important difference is that we avoid spatial aggregation errors by using spatially varying sensitivities at a spatial resolution that degrades with increasing distance from the measurement location. Note that the approach chosen here differs from the above mentioned studies (Michalak et al., 2004; Peylin et al., 2005; Rödenbeck et al., 2003) in that not spatially resolved fluxes are optimized, but rather their spatially resolved sensitivities to the dominant environmental drivers (radiation and temperature) that can after optimization be turned into fluxes.

As a step towards high-resolution retrievals of surface fluxes, we apply this framework to pseudo data generated for the Harvard Forest Environmental Monitoring Site (Barford et al., 2001; Wofsy et al., 1993). The advantage in using pseudo data rather than real data is that we know the truth in the "pseudo-world", so we can control exactly what in- formation we have in terms of the a priori fluxes, and we can estimate how much additional information is provided by the mixing ratio measurements. The two main questions addressed in this paper are: 1) what can we learn, assuming we know prior uncertainty covariance, and 2) what information do we lose, in case we have imperfect knowledge about the prior uncertainty covariance.

In Sect. 2, we present the model linking spatially varying biospheric parameters with $\mathrm{CO}_{2}$ mixing ratios. Section 3 describes the setup of the numerical experiment, including the generation of pseudo data and a priori parameter fields, and retrieval of optimized parameters. Results are presented in Sect. 4 , with the achievable reduction in uncertainty of area averaged fluxes, the spatial resolution of retrievals, and the impact of false assumptions about the length scales of the a priori uncertainty covariance.

\section{Modeling framework}

\subsection{Linking observational constraints to flux model param- eters}

We use the ROAM (receptor oriented atmospheric model) framework described in G03b and by Lin et al. (2004), with STILT as a transport model coupled to surface fluxes on high spatial resolution. Following the notation in Lin et al. (2004), the mixing ratio at the receptor $\mathrm{C}\left(\boldsymbol{x}_{r}, t_{r}\right)$ (with fixed location $\boldsymbol{x}_{r}$ ) can be written as a sum of signals due to surface fluxes $F\left(x_{i}, y_{j}, t_{m}\right)$ (flux at location $\left(x_{i}, y_{j}\right)$ and time $\left.t_{m}\right)$ and contributions from model boundaries $C\left(x_{i}, y_{j}, z_{k}, t_{0}\right)$ :

$$
\begin{gathered}
C\left(\boldsymbol{x}_{r}, t_{r}\right)=\underbrace{\sum_{i, j, m} f\left(\boldsymbol{x}_{r}, t_{r} \mid x_{i}, y_{j}, t_{m}\right) \cdot F\left(x_{i}, y_{j}, t_{m}\right)}_{\text {contribution from sources/sinks }} \\
+\sum_{\text {contribution from advection of upstream tracer field }}^{\sum_{i, k} I\left(\boldsymbol{x}_{r}, t_{r} \mid x_{i}, y_{i}, z_{k}, t_{0}\right) \cdot C\left(x_{i}, y_{j}, z_{k}, t_{0}\right)}
\end{gathered}
$$

Here $f\left(\boldsymbol{x}_{r}, t_{r} \mid x_{i}, y_{j}, t\right)$ is the footprint (also called surface influence), that relates surface fluxes at $x_{i}, y_{j}$, and time $t_{m}$ to mixing ratios at receptors located at $\boldsymbol{x}_{r}$ at time $t_{r}$, and $\left.I \boldsymbol{x}_{r}, t_{r} \mid x_{i}, y_{j}, z_{k}, t\right)$ is the influence (weighting) of concentrations at the boundary. The summations in principle cover the globe, but in practice the domain is much smaller, limited to surface locations and times where $f$ is finite and similarly for $I$. To calculate footprint and influence, STILT was driven by assimilated meteorological data from EDAS (ETA Data Assimilation System) (Rogers et al., 1995) supplemented by GDAS (Global Data Assimilation System) (details see G03b), and run for 15 days backwards in time. The transport simulation was done for the measurement location at Harvard Forest every $3 \mathrm{~h}$ throughout August 2002, resulting in $\mathrm{N}=248$ runs.

Equation (1) can be written using vector notation, with the mixing ratio at the receptor as vector $\boldsymbol{C}$ of dimension $\mathrm{N}$ 
containing the whole time-series $(\mathrm{N}$ discrete points in time $\left.t_{r}\right)$ :

$$
\underbrace{\boldsymbol{C}}_{\text {observations }}=\underbrace{\mathbf{f F}}_{\begin{array}{c}
\text { contribution from surface fluxes } \\
\text { contribution from boundary }
\end{array}}+\underbrace{\boldsymbol{I} \boldsymbol{C}_{b}}_{\text {error }}
$$

Now surface fluxes are presented as modeled fluxes $\boldsymbol{F}$, a vector of length $\mathrm{N}_{F}=\mathrm{N}_{\mathrm{x}} * \mathrm{~N}_{\mathrm{y}} * \mathrm{~N}_{\mathrm{t}}$ containing fluxes at all surface locations and at all times prior to the measurement time, f is a matrix of footprint elements with dimension $\mathrm{N} * \mathrm{~N}_{F}$, and $\mathbf{I}$, the influence matrix relates mixing ratios from the lateral model boundaries $\boldsymbol{C}_{\mathrm{b}}$ to mixing ratios at the receptor. The error term $\boldsymbol{\varepsilon}$ describes differences between measured and modeled values due to uncertainties in transport $(\mathbf{f}, \mathbf{I})$, measurements $(\boldsymbol{C})$, and boundary fields $\left(\boldsymbol{C}_{b}\right)$.

The general idea of the inversion is that information contained in observations can be translated into information about fluxes. Here our focus is on biospheric fluxes $\boldsymbol{F}_{\text {bios }}$ and its dependence on environmental drivers such as light (radiative flux) and temperature. Therefore we write the surface fluxes in Eq. (2) as a sum of biosphere-atmosphere exchange fluxes $\boldsymbol{F}_{\text {bios }}$ and fluxes from combustion processes $\boldsymbol{F}_{\text {comb }}$ (fossil fuel and biomass burning emissions). The biospheric flux representation was simulated using the GSB, with a light and temperature response keyed to vegetation class according to the IGBP vegetation, and with sensitivities derived from fitting to FLUXNET data (Baldocchi and al., 2001) (details see G03b). Here we use a further simplification by only taking into account linear responses to temperature and radiation. Thus the biospheric flux for a given location $l$ and vegetation type $v$ at time $t$ can be written as the sum of gross ecosystem exchange and respiration flux:

$$
\begin{aligned}
& F_{\text {bios }, l, t, v}=G E E_{l, t, v}+R_{l, t, v} \\
& \quad=v c_{l, v}\left(\lambda_{\operatorname{Rad}, l, v} S W R F_{l, t}+\lambda_{\mathrm{Temp}, l, v}\left(T_{l, t}-T_{0}\right)\right) .
\end{aligned}
$$

Here $v c_{l, v}$ is the relative coverage of the given vegetation type in the gridcell at location $l, \lambda_{\mathrm{Rad}, l, v}$ and $\lambda_{\mathrm{Temp}, l, v}$ are the radiation and temperature sensitivities (see also Table 1), $\mathrm{SWRF}_{l, t}$ is the shortwave radiative flux, and $\mathrm{T}_{l, t}$ is the temperature. In vector notation this can be written for all times and locations as

$\boldsymbol{F}_{\text {bios }}=\Phi \lambda$,

where the biospheric flux is written as the product of the parameters vector $\lambda$ (the state vector), that combines the light and temperature sensitivities for different vegetation types at the different flux locations, and matrix $\Phi$, that contains products of relative vegetation cover and temperature or radiation in each grid element at the different times. As in G03b, temperature and shortwave flux are taken from the assimilated meteorological fields used to drive STILT. Rearranging Eq. (2) to separate out the biospheric parameters gives

$\boldsymbol{y}=\mathbf{K} \lambda+\boldsymbol{\varepsilon}_{y}$ with $\boldsymbol{y}=\boldsymbol{C}-\mathbf{I} \boldsymbol{C}_{b}-\mathbf{f} \boldsymbol{F}_{\text {comb }}$ as the observational constraint on biospheric parameters (similar to $\Delta \mathrm{CO}_{2, \text { veg }}$ in $\mathrm{G} 03 \mathrm{~b}$ ), $\mathbf{K}=\mathbf{f} \Phi$ as the Jacobian (sensitivities of measurements $\boldsymbol{y}$ with respect to biospheric parameters $\lambda$ ). Note that the error term $\boldsymbol{\varepsilon}_{y}$ differs from the error term in Eq. (1) in that it also includes errors due to uncertainties in the contribution from combustion fluxes $\mathbf{f} \boldsymbol{F}_{\text {comb }}$ and in the representation of the biospheric fluxes.

By referring to $\boldsymbol{y}=\boldsymbol{C}-\mathbf{I} \boldsymbol{C}_{b}-\mathbf{f} \boldsymbol{F}_{\text {comb }}$ as an observational constraint, although the only observable is the mixing ratio $\boldsymbol{C}$, we made two simplifying assumptions:

1) The contribution from the lateral boundary of the domain $\mathbf{I} \boldsymbol{C}_{b}$ is assumed to be known. Since here we are using simulated measurements rather than real world data, this is not really an assumption. However, in the real world contributions from the boundary can be estimated from a climatological model of background mixing ratios (G03b) or by filtering the measured timeseries itself (Wang, 2003); other possibilities include coupling to a global model or using frequent aircraft data (cf. Fig. 1). In general there will be an estimate of this contribution with an uncertainty that has to be taken into account.

2) Signals from any surface fluxes other than biospheric fluxes, such as fossil fuel emissions of $\mathrm{CO}_{2}$, are assumed to be known. Again, in a world with simulated measurements this is not an issue, however, in the real world this means knowing combustion fluxes $\boldsymbol{F}_{\text {comb }}$ or the signals $\mathbf{f} \boldsymbol{F}_{\text {comb }}$ on small spatial scales well enough, i.e. significantly better than biospheric fluxes or signals. G03b used a combination of measured $\mathrm{CO}$ and emission ratios to estimate fossil fuel and biomass burning signals, but considerable uncertainty is associated with such estimates. Future inversions will need to simultaneously solve for combustion fluxes as well as biospheric parameters, and use of multiple tracers (e.g. CO) may help this task by adding constraints. Here we assume that there are uncertainties associated with fossil fuel and biomass burning emissions that contribute to the overall uncertainty $\boldsymbol{\varepsilon}_{y}$.

Given a set of a priori parameter estimates $\lambda_{\text {prior }}$ with uncertainty covariance $\mathbf{S}_{\text {prior }}$, and following the Bayesian synthesis inversion (a good description of atmospheric inverse methods is given e.g. in Rodgers, 2000), we estimate the state vector $\hat{\lambda}$ as:

$\hat{\lambda}=\left(\mathbf{K}^{T} \mathbf{S}_{\varepsilon}^{-1} \mathbf{K}+\mathbf{S}_{\text {prior }}^{-1}\right)^{-1}\left(\mathbf{K}^{T} \mathbf{S}_{\varepsilon}^{-1} \mathbf{y}+\mathbf{S}_{\text {prior }}^{-1} \lambda_{\text {prior }}\right)$

with error covariance $\mathbf{S}_{\varepsilon}$ for the measurement error $\boldsymbol{\varepsilon}_{y}$. Equation (5) improves the (imperfect) prior knowledge about the parameters $\left(\boldsymbol{\lambda}_{\text {prior }}, \mathbf{S}_{\text {prior }}\right)$ by combining it with the constraint provided by atmospheric measurements $\left(\boldsymbol{y}, \mathbf{S}_{\varepsilon}\right)$ and coupled 
Table 1. Radiation and temperature sensitivity for biospheric fluxes and the associated uncertainties, from fit to AmeriFlux data (after G03b). The last column shows the correlation coefficient between radiation and temperature sensitivity.

\begin{tabular}{llllll}
\hline $\begin{array}{l}\text { Vegetation } \\
\text { type } \\
\text { (simplified) }\end{array}$ & $\lambda_{R}$ & $\sigma\left(\lambda_{R}\right)$ & $\lambda_{T}$ & $\sigma\left(\lambda_{T}\right)$ & $\operatorname{cor}\left(\lambda_{\mathrm{R}}, \lambda_{T}\right)$ \\
\hline & $\mu \mathrm{mol} / \mathrm{m}^{2} / \mathrm{s} /\left(\mathrm{W} / \mathrm{m}^{2}\right)$ & $\mu \mathrm{mol} / \mathrm{m}^{2} / \mathrm{s} /\left(\mathrm{W} / \mathrm{m}^{2}\right)$ & $\mu \mathrm{mol}^{2} / \mathrm{m}^{2} / \mathrm{s} / \mathrm{K}$ & $\mu \mathrm{mol} / \mathrm{m}^{2} / \mathrm{s} / \mathrm{K}$ & -0.74 \\
\hline Forest & -0.018 & 0.013 & 0.14 & 0.11 & -0.72 \\
Cropland & -0.027 & 0.058 & 0.14 & 0.58 & \\
\hline
\end{tabular}

atmospheric transport and biospheric model $\mathbf{K}$. The a posteriori uncertainty for $\hat{\lambda}$ is given by

$\hat{\mathbf{S}}_{\lambda}=\left(\mathbf{K}^{T} \mathbf{S}_{\varepsilon}^{-1} \mathbf{K}+\mathbf{S}_{\text {prior }}^{-1}\right)^{-1}$.

Following Rodgers (2000), we can also write for the posterior estimate of the state vector (Eq. 5):

$\hat{\lambda}-\lambda_{\text {prior }}=\mathbf{A}\left(\lambda-\lambda_{\text {prior }}\right)+\mathbf{G}_{y} \boldsymbol{\varepsilon}_{y}$

with the so called gain matrix $\mathbf{G}_{y}=\left(\mathbf{K}^{T} \mathbf{S}_{\varepsilon}^{-1} \mathbf{K}+\mathbf{S}_{\text {prior }}^{-1}\right)^{-1} \mathbf{K}^{T} \mathbf{S}_{\varepsilon}^{-1}$ and the averaging kernel matrix $\mathbf{A}=\mathbf{G}_{y} \mathbf{K}$. In Eq. (7) we can interpret a given column of $\mathbf{A}$ as the response of the retrieval to a perturbation of the corresponding element of the state vector. This will be used in Sect. 4.2.

\subsection{Reduction in dimension of state space}

The simplicity of Eq. (4) hides the complexity and magnitude of the problem: the representation links light and temperature sensitivities $\lambda$ of biospheric fluxes (the state vector) from different vegetation types at all times and all surface flux locations that influence the measurements to an observable constraint $\boldsymbol{y}$. For example, for a spatial domain that roughly covers North America at a resolution of $20 \mathrm{~km}$ there are 120000 different gridcells, and about $1 / 4$ of these cells are covered by vegetation. For two vegetation types and two sensitivities (to light and temperature), each of these 30000 vegetated cells requires 4 degrees of freedom at any given time; and if one is interested in e.g. a one-month duration and allows the sensitivities to vary on daily timescales, the required number of parameters is about 3.6 million. A retrieval on a grid with such a number of unknowns is not feasible - e.g., the prior uncertainty covariance matrix itself will have more than $10^{13}$ elements, and plainly there is insufficient information in the data to constrain so many parameters.

Therefore it is necessary to reduce the state space dimension significantly (both spatially and temporally), ideally without losing any information. We know that for a given measurement location the spatial differences in surface fluxes

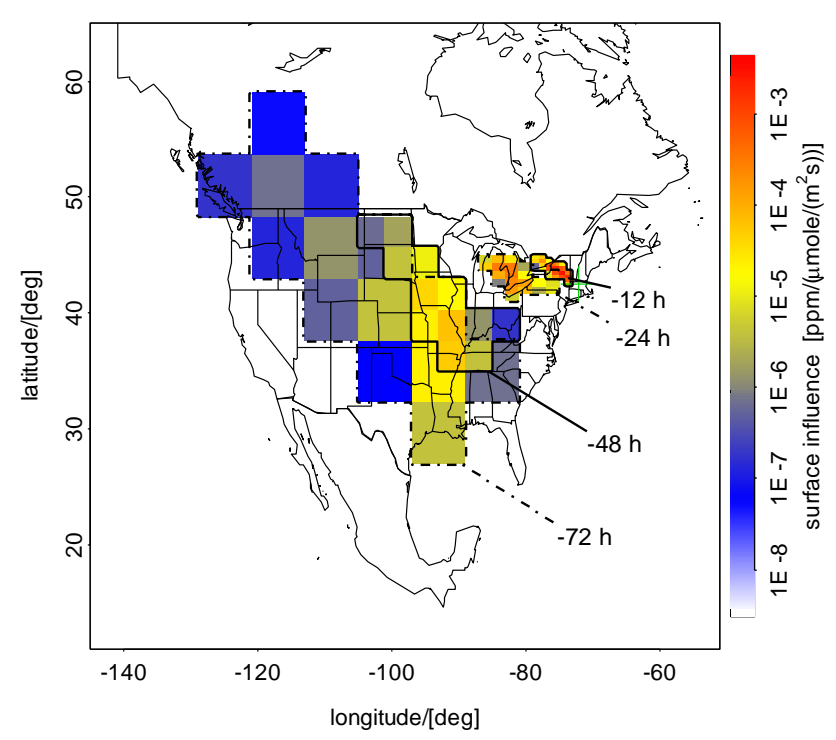

Fig. 2. STILT derived footprint for the Harvard Forest Monitoring Site on 17 August 2002 at 15:00 GMT, for different times backwards and integrated over $3 \mathrm{~h}$ (i.e. $-12 \mathrm{~h}$ denotes the interval from 12 to $15 \mathrm{~h}$ prior to the release time). As the area of the footprint at a given time increases, the dynamic resolution from STILT chooses coarser pixels to represent the footprint. The footprints for different resolutions are rescaled to the finest resolution grid $\left(1 / 6^{\circ}\right.$ lat $\times 1 / 4^{\circ}$ lon $)$ to avoid the trivial dependence on grid area.

at some far away small surface area does not affect local mixing ratios and need not be (indeed, cannot be) accounted for separately from surrounding surface elements. The reason for this is that the size of the footprint $\mathrm{f}\left(\boldsymbol{x}_{r}, t_{r} \mid \boldsymbol{x}, t\right)$ for a measurement made at location $\boldsymbol{x}_{r}$ and time $t_{r}$ increases with elapsed time $t_{r}-t$ due to atmospheric mixing, and the magnitude of the elements of $f$ correspondingly decrease, as illustrated in Fig. 2. The footprint-area at 3 days prior $(-72 \mathrm{~h})$ is more than 10 times larger than the footprint-area at 1 day prior to arrival. Figure 2 also illustrates our approach to enlarge the grid size with increasing footprint area, "dynamic grid resolution" as described in G03b. This dynamic resolution was necessary due to the limited number of particles 


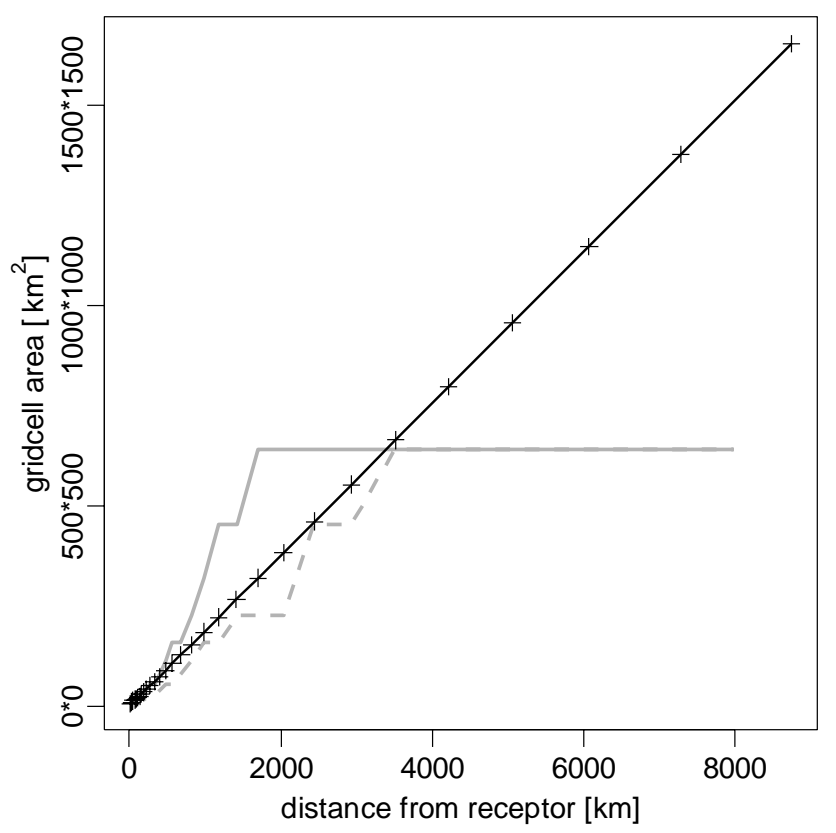

Fig. 3. Gridcell area vs. distance from the receptor location. The dynamic resolution from STILT is shown as solid gray line (50\%ile of influence) and dotted gray line (10\%ile of influence). The solid black line indicates the spatial resolution of the polar projection with 32 sectors and 30 radial segments, with each symbol representing a radial segment. Note that the square root of the gridcell area is plotted as y-axis.

representing the airmass. Results using high resolution footprints throughout $(20 \mathrm{~km}$ up to 15 days, with 4000 particles) rather than dynamically adjusted resolution (also with 4000 particles) showed only minor differences (rms error $\sim 3 \%$ of the biosphere signal, or a factor of 4 smaller than the uncertainty due to the limited number of 100 particles representing the airmass, see G03b). From this we can conclude that spatial variations in surface fluxes on scales smaller than the resolved scale at a given time (with dynamic grid resolution) have no significant impact on mixing ratios at the receptor.

Here we need a grid with a resolution that decreases with distance from the receptor, and that does not change with time, thus defining an appropriate state space for a given receptor. Hence we use a simple polar projection, with $32 \mathrm{sec}-$ tors and 30 radial discs to represent the footprints. The grid was chosen so that the area for each grid-cell as a function of distance from the receptor $r$ behaves similar to the dynamic grid (Fig. 3), with a grid cell area increasing proportional to $r^{2}$, increasing the radial step-size proportional to $r$, starting with a minimum step-size of $20 \mathrm{~km}$. Since the dynamic grid resolution changes (in time) for a given $r$, the $10 \%$ ile (50\%ile) of the gridcell area is shown in Fig. 3, i.e. $10 \%$ $(50 \%)$ of the influence was represented with a higher resolution, respectively. The grid-cell area of the chosen polar

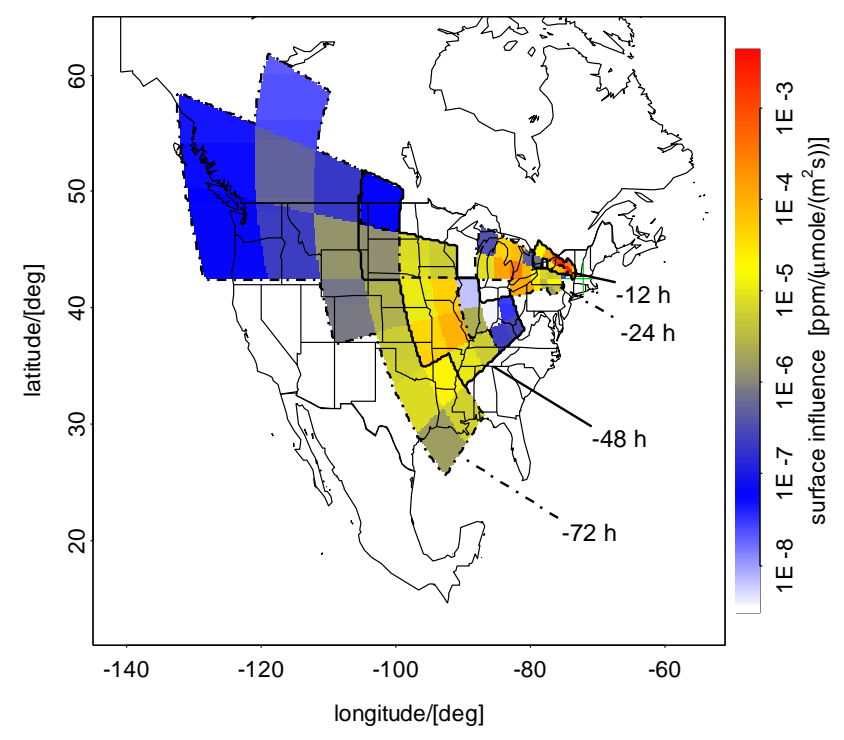

Fig. 4. Same footprint as Fig. 2, but represented using polar coordinates.

grid representation falls between the $10 \%$ ile and the $50 \%$ ile curve of the dynamic grid, i.e. has appropriate resolution in far more than $50 \%$, but not quite $90 \%$ of the time. As an example the footprints shown in Fig. 2 are recalculated in polar coordinates, and than transformed back to Cartesian coordinates for visualization (Fig. 4). The relevant spatial structure in the footprint is well represented on the polar coordinates.

The parameters $\lambda$ are allowed to vary spatially, with resolution given by the polar grid, reducing the size of the state vector $\lambda$ by a factor of about 60 compared to the Cartesian grid (Lat-Lon), without significant loss of information. Since we are here interested primarily in the spatial scales, we made the simplifying assumption that the parameters $\lambda$ are constant over one month, reducing the state space dimension by another factor of 30 compared to daily varying parameters. This choice thus makes a retrieval of the remaining 2048 elements of $\lambda$ feasible.

\section{Experiment setup}

The experiment consists of the following steps:

1) Pseudo data are generated from a set of parameter fields (the "truth", Sect. 3.1).

2) A number of a priori parameter fields are generated, with spatial covariance decaying with different decorrelation length scales (and encapsulated in the uncertainty covariance matrix) (Sect. 3.2).

3) A posteriori parameter fields are retrieved based on the prior and an assumed prior uncertainty covariance which may differ in its decorrelation length scale from the true uncertainty covariance of the prior (Sect.3.3). 
4) Fluxes based on the retrieved and the true parameter fields are calculated for different scales of spatial averaging (Sect. 3.4).

\subsection{Generation of pseudo data}

Pseudo data for biospheric signals $\boldsymbol{y}_{t}$ are calculated after Eq. (2) from "true" parameter fields $\lambda_{t}$ that are assumed to be spatially constant for simplicity (spatially uniform light and temperature sensitivity for forests and croplands after Table 1). Even though the parameters fields are spatially constant, strong gradients in surface fluxes result from the different responses for different vegetation types, and from gradients in the environmental drivers temperature and radiation. A realization of the error term $\boldsymbol{\varepsilon}_{y}$ was created based on a Gaussian sample with standard deviation of $2 \mathrm{ppm}$. This uncertainty is meant to account for (a) uncertainty in the measurements themselves (less than $0.5 \mathrm{ppm}$ at Harvard Forest), and errors in the modeling framework such as (b) inability to reproduce eddies in the atmosphere (ranging from 0.2 to $2 \mathrm{ppm}-\mathrm{G} 03 \mathrm{a}$ ), (c) uncertainties in fossil fuel signals (order of ppm-G03a), (d) uncertainties in the tracer boundary fields ( $\sim 1$ ppm after G03a), and (e) errors in the biospheric model formulation (true fluxes are affected by factors other than temperature and radiation). The $2 \mathrm{ppm}$ assumed uncertainty is a lower limit, or the information content of the measurements derived here represents an upper bound, since uncertainties related to imperfect transport are not included. The transport error for the current framework is on the order of 2 ppm for the Harvard Forest site, following methods discussed in Lin and Gerbig (2005); however, we did not include this since it can be assumed that in future models this will improve.

The simulated $\mathrm{CO}_{2}$ signals using values for the biosphere flux parameters from G03b (not including the measurement error) are shown in Fig. 5, together with observed $\mathrm{CO}_{2}$. The simulations have a lot of similarity with the measurements, with a pronounced diurnal and synoptic variability. Diurnal maxima are not well represented due to the inability of the model to reproduce a shallow nocturnal boundary layer: the modeled boundary layer height is often too high, causing the footprint to cover a larger area (due to larger wind speeds in the deeper layer) and the biospheric signal from nocturnal respiration to be too small (due to the vertical dilution in a deeper layer). This underestimation of respiration signals in the pseudo data means that processes dominating the dayand night-time fluxes (assimilation and respiration) will not be as separable as in the real world. Also shown in Fig. 5 are the simulated $\mathrm{CO}_{2}$ signals using the Cartesian grid, nearly indistinguishable from the version based on the polar grid (standard deviation of differences $0.6 \mathrm{ppm}$ ), supporting our claim that no significant loss of information occurred in the regridding from Cartesian to Polar coordinates.

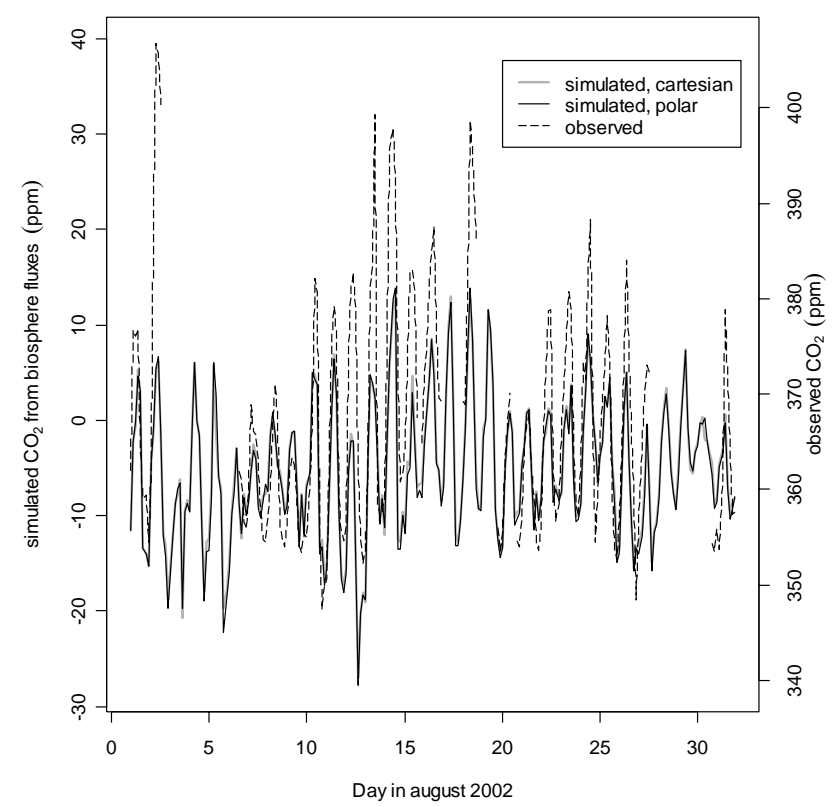

Fig. 5. Simulated $\mathrm{CO}_{2}$ mixing ratios at Harvard Forest from biospheric fluxes using dynamic gridsize (gray line), and polar grid (thin black line). Also shown as dashed line are observations on the same scale, shifted for better readability (right vertical axis).

\subsection{Generation of a priori parameter fields}

A priori parameter fields are created by adding noise to the "true" parameter values, with the same variance as the prior uncertainty (Table 1), and with a variety of decorrelation length scales $l_{\text {true }}$ ranging from 10 to $15000 \mathrm{~km}$. The spatial covariance was assumed to decay exponentially with distance. Thus a covariance matrix was constructed with diagonal elements as prior uncertainties (from G03b), and offdiagonal elements decaying with $\exp \left(-d / l_{\text {true }}\right)$, with $d$ as the distance between the grid cells. Parameter fields were then generated as a realization of the noise using spectral decomposition of this covariance matrix (see e.g. Cressie, 1993).

\subsection{Retrieval of parameter fields}

Posterior fluxes and associated uncertainties are retrieved from the pseudo data and the prior parameter fields following Eq. (5). As mentioned before, the decorrelation length scale for the assumed prior error $(l)$ can be different from the one used to create the prior parameter field $\left(l_{\text {true }}\right)$, since in the real world this length scale is not necessarily known. Also, the aim of this work is to test how results depend on this knowledge. Therefore fluxes are retrieved for $9 * 9$ different combinations of assumed prior decorrelation scale $l$ and true decorrelation scale $l_{\text {true }}$. To allow statistical sampling of these retrievals, 500 different realizations of prior parameter fields were used, resulting in a total number of 40500 retrievals. 
average footprint August 2002: Day

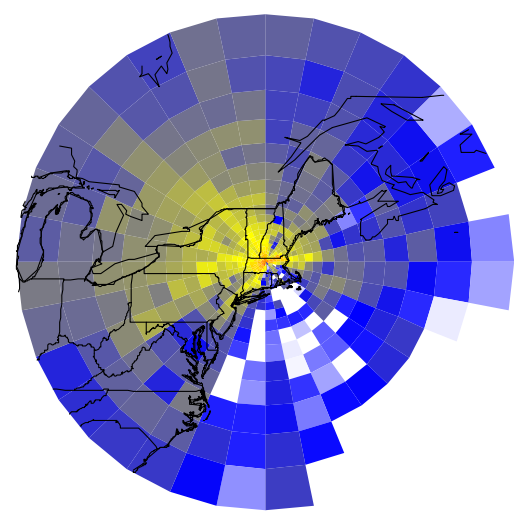

Forest coverage

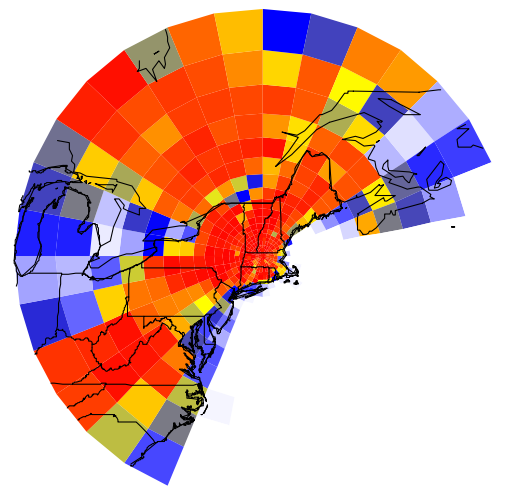

Temperature 8/24/04, 18 GMT

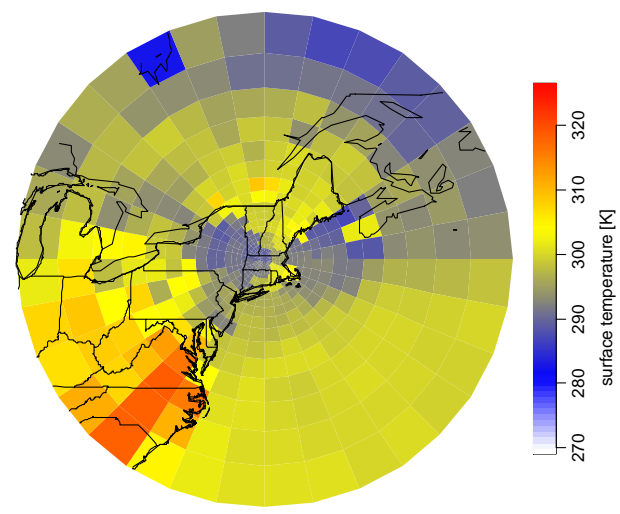

average footprint August 2002: Night

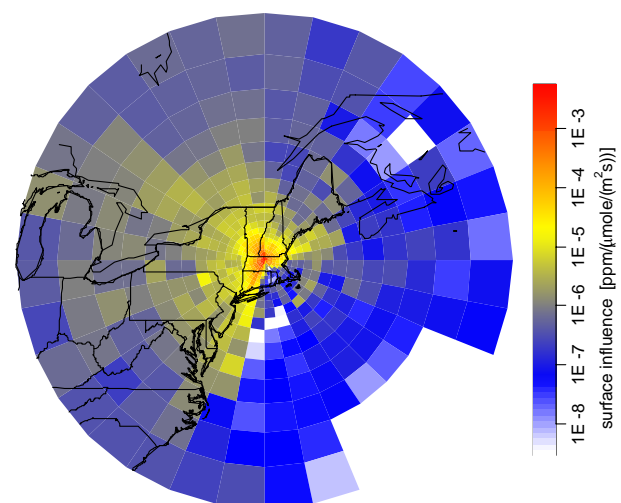

Cropland coverage

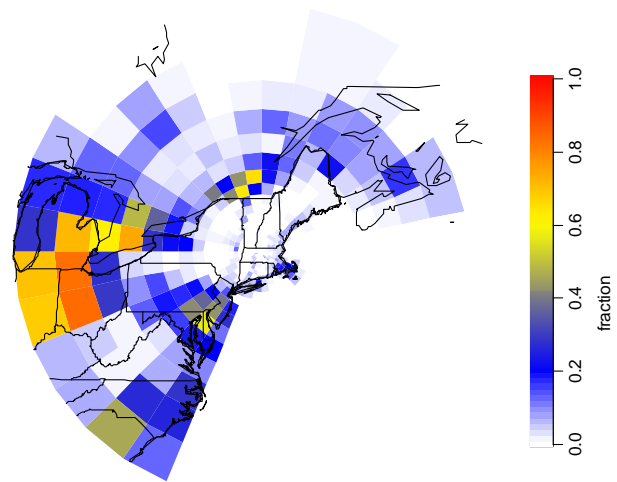

Radiation 8/24/04, 18 GMT

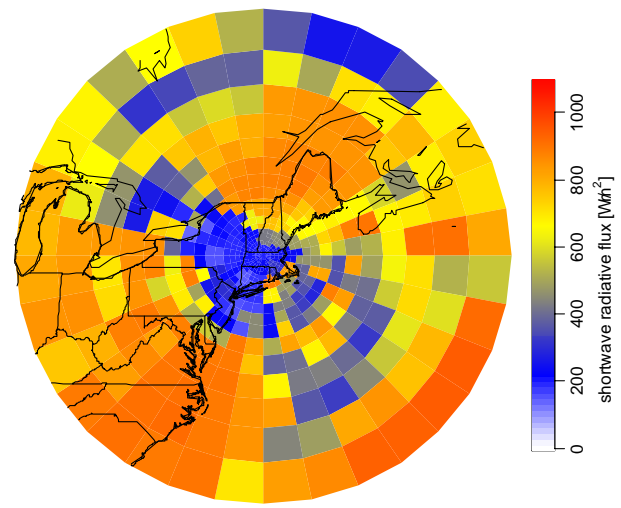

Fig. 6. Monthly averaged footprint for Harvard Forest daytime (18:00 and 21:00 UT, top left) and nighttime measurements (06:00 and 09:00 UT, top right), as well as vegetation coverage (bottom). The map is centered around the Harvard Forest Monitoring Site with a radius of $1500 \mathrm{~km}$. Note that the footprint maps are rescaled to a $20 \times 20 \mathrm{~km}$ grid for comparability with the cartesian grid and to avoid the trivial dependence on grid area.

\subsection{Area averaging}

Results are presented in terms of retrieved fluxes and their uncertainties for averages over areas of different sizes ranging from local to continental scales. Ideally, flux aver- aging should be done over areas where the footprint is strongest, such as in the dominant wind direction upwind of the receptor (see Fig. 6, top). However, for simplicity we use circular areas centered at the Harvard Forest site with radii of 100,514 and $1849 \mathrm{~km}$. In fact, the footprint distribution 
reduction in uncertainty GEE forest

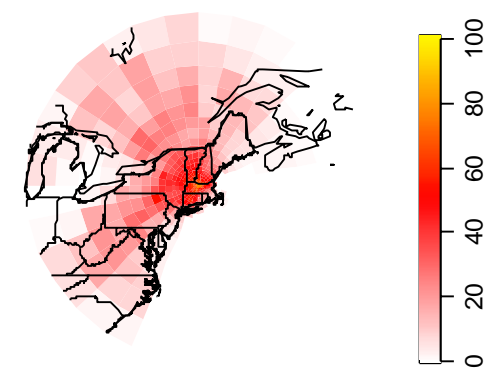

reduction in uncertainty $R$ forest

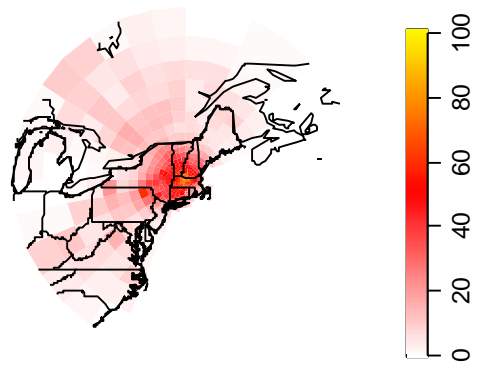

reduction in uncertainty GEE crop

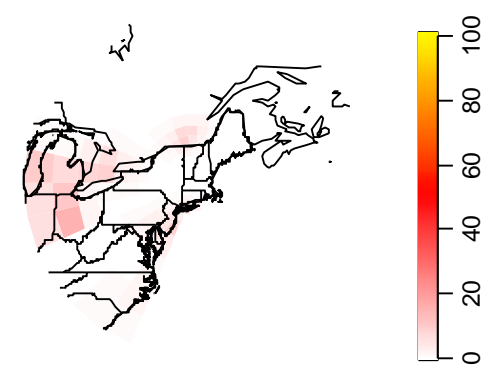

reduction in uncertainty $R$ crop

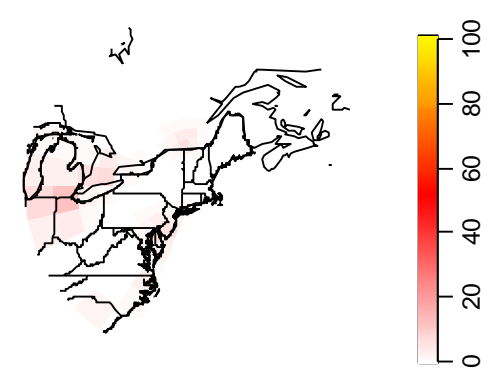

Fig. 7. Uncertainty reduction (in \%) for $100 \mathrm{~km}$ prior uncertainty correlation scale for the different parameters within the state vector. Left column is for forest, right for crop, top row for light sensitivity (controlling GEE), and bottom row for temperature (controlling R). The map is centered around the Harvard Forest Monitoring Site with a radius of $1500 \mathrm{~km}$.

seems to coincide with the land/ocean distribution, with low influence from the ocean. Given the focus on fluxes from the terrestrial biosphere, the simple circular averaging seems appropriate. To transform the state vector $\lambda$ (spatially resolved light and temperature sensitivities for forests and cropland) into area and time averaged fluxes, we define the aggregation operator $\mathbf{W}$ :

$F=\mathbf{W} \boldsymbol{F}=\mathbf{W} \Phi \lambda$.

Here $F$ is the area-averaged flux (scalar) on the polar grid, $\boldsymbol{F}$ is the spatially resolved flux, and $\Phi$ contains the time averaged radiation and temperature distribution to convert the state vector into fluxes as defined in Sect. 2.1. The spatial distribution of vegetation type (forest and crop) and an example of the drivers (radiation and temperature) for a specific time are also given in Fig. 6. The error covariance matrices $\mathbf{S}_{\lambda}$ have to be transformed accordingly (Rodgers, 2000):

$S_{F}=\mathbf{W S}_{\boldsymbol{F}} \mathbf{W}^{t}=\mathbf{W} \Phi \mathbf{S}_{\lambda} \Phi^{t} \mathbf{W}^{t}$

$\mathbf{S}_{\boldsymbol{F}}$ is the covariance matrix for the spatially resolved flux, and $S_{F}$ is the variance of the area-averaged flux (a scalar).

\section{Results}

The results of the numerical experiment are presented in the following sections related to the two main questions:

- what can we learn, assuming we know prior uncertainty covariance (i.e. with $l=l_{\text {true }}$ ), and

- what information can we lose, in case we don't exactly know the prior uncertainty covariance (i.e. with $l \neq l_{\text {true }}$ ).

The first question is addressed in Sects. 4.1 and 4.2, while Sect. 4.3 investigates the extent to which we retrieve false information by assuming the wrong a priori uncertainty covariance length scale.

\subsection{Uncertainty reduction}

By combining the prior information about the state vector $\lambda$ with the additional constraint posed by the pseudo measurements, the uncertainty in the retrieved state vector $\hat{\lambda}$, expressed by the matrix $\hat{\mathbf{S}}_{\lambda}$, is expected to be reduced compared to the prior uncertainty (matrix $\hat{\mathbf{S}}_{\text {prior }}$ ). The reduction 
cov. scale $10 \mathrm{~km}$

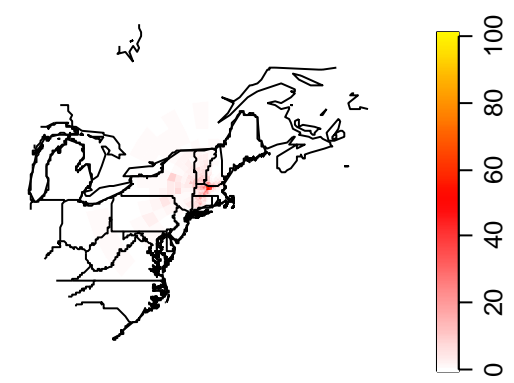

cov. scale $1000 \mathrm{~km}$

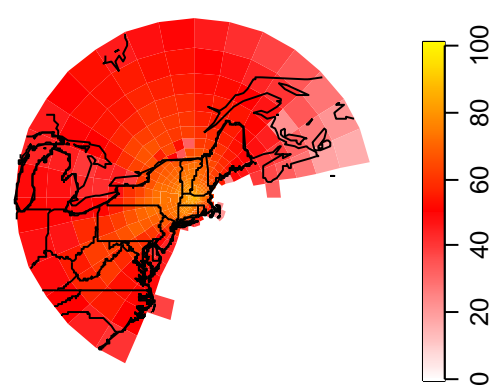

cov. scale $100 \mathrm{~km}$

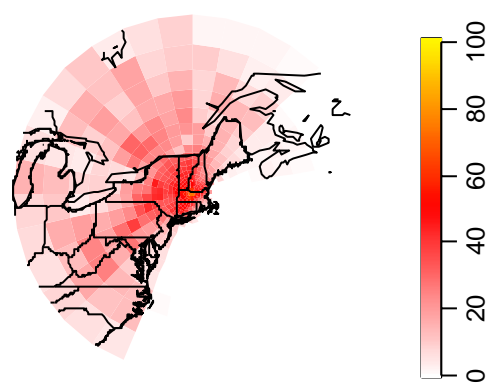

cov. scale $15000 \mathrm{~km}$

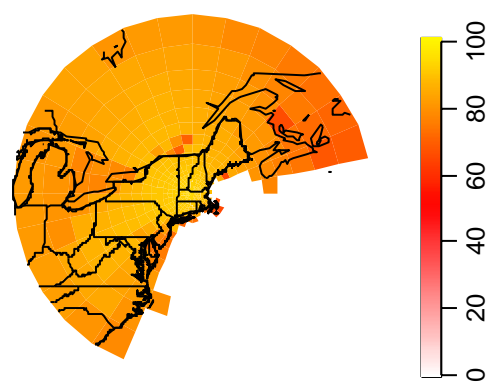

Fig. 8. Uncertainty reduction (in \%) in the spatially resolved flux (averaged over the month of August 2002) for different prior uncertainty correlation scales. The map is centered around the Harvard Forest Monitoring Site with a radius of $1500 \mathrm{~km}$.

of the uncertainty for the individual elements of $\lambda$ can be expressed as $1-\sigma_{\text {posterior }} / \sigma_{\text {prior }}$, where the $\sigma$ 's are the square root of the diagonal elements of the corresponding covariance matrices. The uncertainty reductions for the different elements of the state vector are shown in Fig. 7. Most of the information contained in the measurement relates to the near field of measurement location, and the information content decays with increasing distance. This is related to the behavior of the footprint, which decays strongly with increasing distance from the receptor. Respiration affects concentrations most directly at night, when advection is weak, hence the information about $\mathrm{R}$ is spatially more concentrated than about GEE. Since there is no cropland vegetation in the near field of Harvard Forest (see middle right graph of Fig. 6), there is no local uncertainty reduction for the corresponding light and temperature sensitivity. The nearest significant cropland influence on the mixing ratios is in the Midwest, for which there is a reduction in uncertainty of about $20 \%$ and $10 \%$ for the light and temperature sensitivity, respectively.
The resulting reduction of uncertainty for fluxes is shown in Fig. 8 for a range of prior uncertainty correlation scales $l_{\text {true }}$ (with $l=l_{\text {true }}$ ). It is obvious that the spatial scale of retrieved information directly reflects the prior uncertainty correlation scale. For short $l$ only a small uncertainty reduction is attained in the very near field, while for large $l$ an uncertainty reduction of nearly $100 \%$ is obtained for a large area surrounding the measurement location. This strong dependence on the a priori uncertainty covariance shows that plots of uncertainty reduction are not very useful indicators of the potential constraint of an observational site on surface fluxes, that is without making a disclaimer about the assumed a priori uncertainty covariance length scale.

Uncertainties in fluxes for the different averaging scales (areas with different radii around Harvard forest) are shown in Fig. 9. The prior uncertainty becomes smaller with decreasing $l$, as expected for an average of more and more statistically uncorrelated regions. As $l$ increases beyond the averaging scale for the flux (indicated by the arrows), the resulting prior uncertainty for the averaged flux starts saturating (e.g. for $516 \mathrm{~km}$ averaging scale at about $1.5 \mu \mathrm{mol} / \mathrm{m}^{2} / \mathrm{s}$ ). 
The reason for this saturation is that for $l$ large compared to the averaging scale there is only a single degree of freedom in each parameter, independent of the exact size of $l$.

As expected, the posterior uncertainties are significantly smaller than the a priori uncertainties, with an uncertainty reduction exceeding $50 \%$ for prior uncertainty correlation scales $l$ larger than $300 \mathrm{~km}$. The behavior of prior uncertainty for area averaged fluxes with $l$ (decreasing with decreasing $l$ ) is partly compensated by the opposite behavior of the uncertainty reduction. Thus the resulting posterior uncertainty for area averaged fluxes shows a maximum near the averaging scale. In different words, for small $l$ we already have a lot of prior knowledge about the flux (many independent errors in small regions), and the information from the atmosphere doesn't help that much, while for large $l$ there are effectively only a few degrees of freedom that can easily be constrained by atmospheric observations.

4.2 Averaging kernel: How sharply can a tower see parameter fields?

Here we investigate how local changes in the parameter field can be resolved with the retrieval based on the combination of $\mathrm{CO}_{2}$ measurements and a set of a priori parameters. As introduced at the end of Sect. 2.1, a given column of the averaging kernel matrix $\mathbf{A}$ describes the retrieval of a perturbation of the corresponding state vector element (see Eq. 7). An example for this is shown in Fig. 10, where the light sensitivity for forest is perturbed at the gridcell located at a distance of $50 \mathrm{~km}$ to the south-west of Harvard Forest. A perturbation of the size of the a priori uncertainty of the parameter was applied, given by the square root of the corresponding diagonal element in the prior uncertainty covariance matrix. The map presented in Fig. 10 shows the corresponding retrieved flux due to the change of light and temperature sensitivity for forest in the proximity of Harvard Forest, weighted by forest coverage.

Ideally, the Averaging kernel would be a diagonal matrix, and the retrieval would only show a response at the location of the perturbation. However, the loss of information due to atmospheric mixing causes the response to be spread out around the exact location. In addition, the length scale of the a priori uncertainty covariance $l$ also influences the spreading of the response. The retrieval suggests also a significant change in temperature sensitivity for a perturbation in the light sensitivity ("cross-talk"), this is related to the fact that $\mathrm{CO}_{2}$ signals caused by respiration (temperature sensitivity) and by assimilation (light sensitivity) are not completely independent. In fact the retrieved flux due to cross-talk (top right of Fig. 10) shows a response that varies with distance from the tower, with maximum values that are even larger than the direct response (top left of Fig. 10). This is related to the much larger posterior uncertainty in the temperature sensitivity (about a factor 7 when expressed in fluxes). The situation is different for a perturbation in temperature sensi-

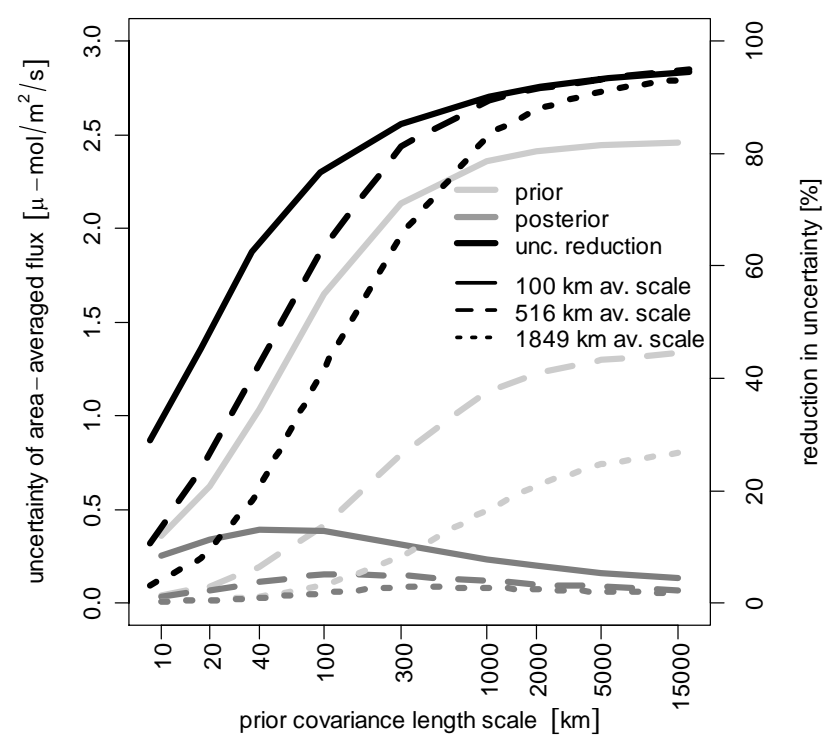

Fig. 9. Prior (light grey) and posterior uncertainties (medium grey) vs. length scale of prior error covariance $\left(l=l_{\text {true }}\right)$ for averaging scales of $100 \mathrm{~km}$ (continuous lines), $516 \mathrm{~km}$ (dashed lines) and $1849 \mathrm{~km}$ (dotted lines). Also shown is the reduction in uncertainty (black lines, right vertical scale).

tivity (bottom of Fig. 10): the cross-talk is minimal, the maximum of the retrieved flux is located correctly, but there is a large flux close to the tower with opposite sign. In addition there is also some cross-talk between forest and crop sensitivities (not shown). These results indicate that the method is capable of retrieving local changes in the parameter field, but not without some aliasing, both spatially and (as crosstalk) between the temperature and radiation sensitivity. The cross talk is principally not avoidable; it is related to the fact that the measured $\mathrm{CO}_{2}$ signal does not contain the full information about the different sensitivities at the different locations. The magnitude of the cross-talk depends on the a priori knowledge: If the a priori knowledge about respiration fluxes (i.e. temperature sensitivity) would be comparable to that about uptake fluxes (light sensitivity), there would be less cross-talk from light to temperature sensitivity, but more cross-talk in the other direction.

\subsection{The case of unknown correlation scale for prior uncer- tainty}

The above results refer to the reduction in uncertainty that can be achieved if we know $l_{\text {true }}$, the true prior uncertainty correlation scale. Now we investigate what happens when it is not known, i.e. when $l \neq l_{\text {true }}$. Figure 11 shows the ratio of the standard deviation of the 500 flux retrievals (based on different realizations of the a priori parameter fields for a given $l_{\text {true }}$ ), to the assumed posterior uncertainty (based on an assumed prior uncertainty correlation scale $l$ ), for an averaging scale of $516 \mathrm{~km}$ (radius of the circular area around Harvard 

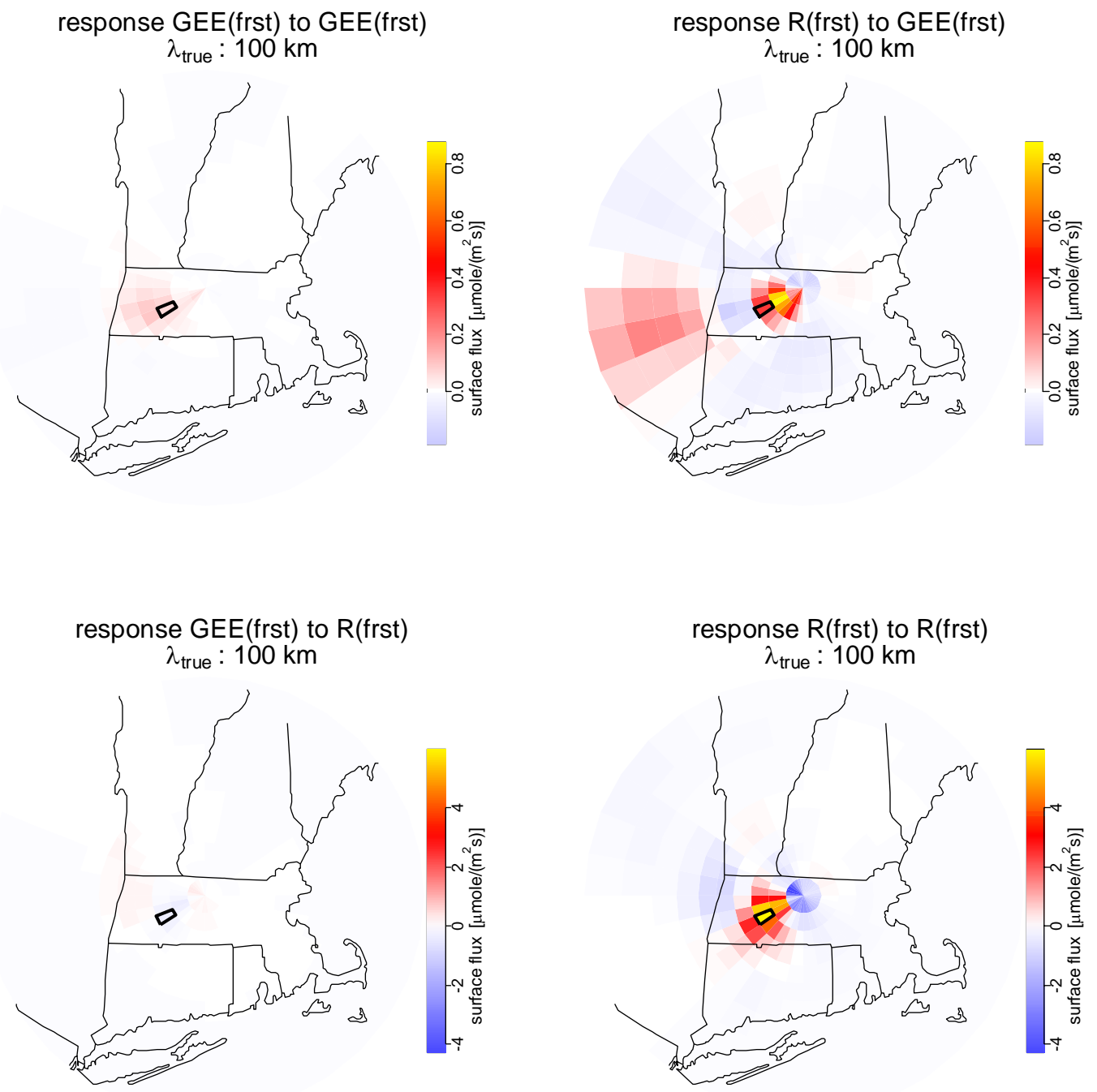

Fig. 10. Response of the retrieval in flux units (left: forest light sensitivity, right: forest temperature sensitivity) to a local perturbation of the size of the a priori uncertainty in the parameter field at the gridcell $50 \mathrm{~km}$ to the $\mathrm{SW}$ of Harvard Forest (top: perturbation in forest light sensitivity, bottom: perturbation in forest temperature sensitivity). Note the difference in scale between top and bottom.

Forest). If this ratio of actual over assumed uncertainty (i.e. the uncertainty underestimation), is larger than one, a false reduction of uncertainty is the result. For example, if the true scale $l_{\text {true }}$ is $100 \mathrm{~km}$ (the gray curve in Fig. 11 with the filled circle at $100 \mathrm{~km}$ ), and the assumed scale $l$ is $10 \mathrm{~km}$, the derived posterior uncertainty is a factor of 10 too small (value of the curve at $10 \mathrm{~km}$ ). In this situation the true flux would not fall within the range given by a single retrieval and the assumed uncertainty estimate, but can only be expected to be within a $10-\sigma$ range around the estimate. Similarly, if in the same case $\left(l_{\text {true }}=100 \mathrm{~km}\right)$ we assume a scale $l$ of $15000 \mathrm{~km}$, the uncertainty is underestimated by a factor of 4 . On the other hand, for a scale $l_{\text {true }}$ of $10 \mathrm{~km}$, the uncertainty is overestimated for assumed scales smaller than about $1000 \mathrm{~km}$. In these cases of uncertainty overestimation the true flux will fall within the error bars given by the posterior uncertainty, but would be much closer to the value than indicated by the posterior uncertainty, thus information would be unused.

Experiments with different averaging scales $(100 \mathrm{~km}$ and $1849 \mathrm{~km}$ circle around Harvard Forest, not shown) generally show a similar behavior: if the prior uncertainty covariance scale $l_{\text {true }}$ is shorter than the averaging scale, and if $l_{\text {true }}$ is underestimated $\left(l<l_{\text {true }}\right)$, the posterior uncertainty is more and more underestimated. This is related to the significant underestimation of the prior uncertainty: by assuming a too small decorrelation scale, the many uncorrelated errors in the prior cancel out as $1 / \sqrt{N}$, with $N$ as the number of independent degrees of freedom within the averaging area. Similarly, if the prior uncertainty covariance scale $l_{\text {true }}$ is larger than the averaging scale, and if $l_{\text {true }}$ is overestimated $\left(l>l_{\text {true }}\right)$, the 
posterior uncertainty is also more and more underestimated. This effect is similar to the aggregation error: a too large correlation scale in the prior serves as an aggregation of the information into only a few degrees of freedom (a few large scale bias errors).

\section{Discussion and outlook}

In this experiment we have addressed the issue of aggregation errors, which occur when inhomogeneous sampling covaries with fluxes that cannot occur within the statistical model. The choice of inhomogeneous spatial grid with high resolution in the near-field of the measurement for biosphereatmosphere exchange largely avoids the aggregation error in its classical sense (Kaminski et al., 2001). A spatial aggregation error is reintroduced in the pseudo experiment when using a too large covariance length scale for the a priori error; this represents a reduction of the effective degrees of freedom. Further, temporal aggregation error is largely reduced by choosing a representation of biosphere fluxes in a very simple diagnostic model, with parameters that control response to light and temperature and thus allow for diurnally varying fluxes. When applying such a model to the real world, however, it has to be noted that the a priori uncertainty $\mathbf{S}_{\text {prior }}$ as well as the measurement error $\mathbf{S}_{\varepsilon}$, did not include temporal correlation. The same basic principles apply for these temporal correlations as for the investigated spatial correlations, in that the interplay of averaging time scales, integrating time scales of the atmosphere, and the differences in true and assumed temporal correlations can cause biased results or loss of information. Also structural error due to inadequate model parameterization (e.g. changes of light sensitivity with water availability) has not been taken into account; so for real world applications the model either needs to be able to resolve resulting variations in fluxes, or it has to be allowed for in a corresponding uncertainty with the correct spatial and temporal covariances.

The obtained results can be used to address an important question related to regional carbon budgets: how many observing stations are required for a given accuracy of the regional budget estimate. A rough approximation for the achievable accuracy of a flux retrieval based on a network of tall towers can be based on the range of posteriori uncertainties (0.001-0.023 $\mu$ moles $/ \mathrm{m}^{2} / \mathrm{s}$ from Fig. 9) for an area of $516 \mathrm{~km}$ radius around each tower: with 10 towers the flux of an area of the size of the contiguous United States could be determined with an accuracy of 0.14-2.8 Mt C/year (assuming that the uncertainties are uncorrelated between the different circles around the sites). In comparison, current global scale inversions achieve a posterior uncertainty for monthly fluxes of the order of $1 \mathrm{Gt}$ C/year for temperate North America (Gurney et al., 2004).

Although in this pseudo experiment we have paid attention to using appropriate magnitudes for the various error terms,

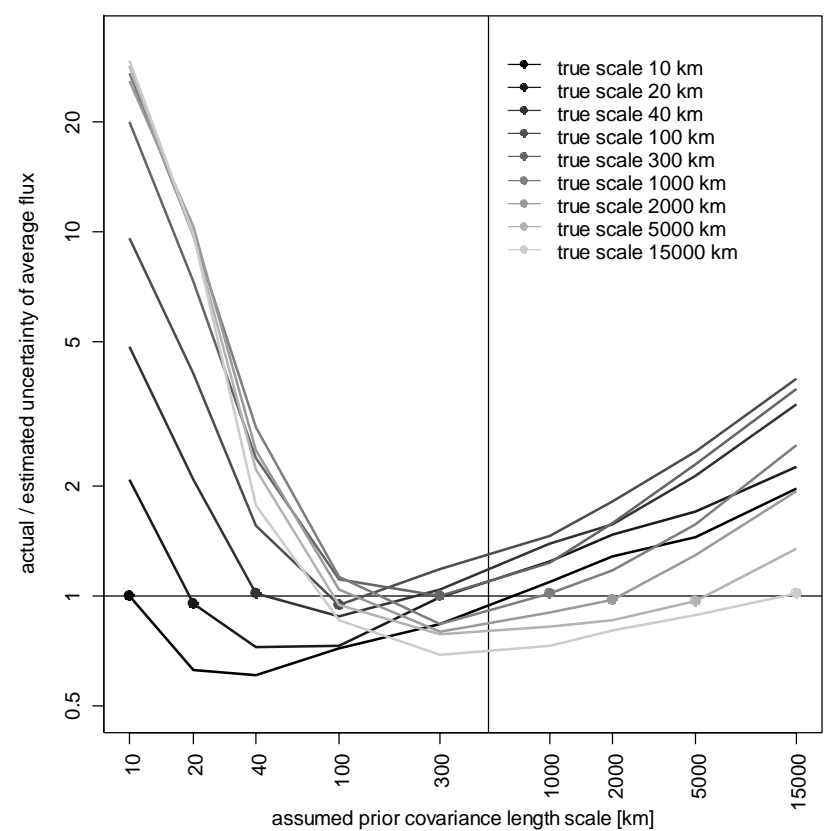

Fig. 11. Ratio of actual to estimated posterior uncertainty of flux average plotted against assumed prior covariance scale $l$. The different lines correspond to different $l_{\text {true }}$. (used for creation of prior realizations), and points are plotted for $l=l_{\text {true }}$. The vertical bar indicates the scale for flux averaging $(516 \mathrm{~km})$. Values $>1$ indicate that the retrieval produces an erroneous underestimation of uncertainty in the posterior parameter values.

there is certainly additional uncertainty in the representation of biospheric fluxes with simple temperature and light sensitivities, also vertical transport remains uncertain, with potential biases. Further, the assumption of a covariance matrix with an exponentially decaying spatial correlation might not be appropriate. However, we do not feel that there is room for more than an order of magnitude in the posterior uncertainty. Given the 2-3 orders of magnitude larger uncertainties from coarse global inversions, this clearly shows the potential of high resolution transport modelling coupled to diagnostic biosphere modelling, and using continuous records to solve for parameters in the biospheric model. Of course this potential has yet to be realized in the future by fully coupling the regional to the global scales.

The pseudo experiment also shows that the usefulness of information from atmospheric trace gas observations depends critically on what we know a priori about the flux distribution. Of course a large contribution to the reduction achieved in the posterior uncertainty is from the assumptions in the prior, e.g. for a very short correlation length scale of $10 \mathrm{~km}$ for the a priori uncertainty, the flux of the US would already be determined down to an uncertainty of $0.17 \mathrm{MtC} /$ year, and not much is added by the network. In other words, the number of stations needed to constrain the flux at a given resolution over the whole continent is directly 
related to the reduction of uncertainty presented in the maps of Fig. 8. With a very short a priori uncertainty correlation length scale, the information gain only extends over small patches and reaches, apart from the next proximity of the tower, less than $20 \%$. The reduction of uncertainty is further modulated by the tracks of the air masses on their way to the tower. In the case presented here these footprints were accumulated over a month, so if shorter periods are required, the regions with information gain are limited to even fewer areas. On the other hand, for very large scales, a single station is sufficient to constrain a large area, and the exact location of the footprints is less important. So the dominant scale for determining the network density is the scale over which the priori uncertainty in the parameters (or in the fluxes, if one solves for fluxes) correlates; the scale of variations in the footprint becomes important at shorter decorrelation scales. Similarly, the scale with which local perturbations in the behavior of the biosphere can be resolved depends primarily on the scale of prior error covariance, as plots similar to Fig. 10 but different $\boldsymbol{l}_{\text {true }}$ indicate (not shown).

It is important to note that all errors were assumed Gaussian. When applying such a method to other trace gases, such as $\mathrm{CO}$, this assumption is not valid: the distribution of $\mathrm{CO}$ in the atmosphere is lognormal, mostly caused by the interaction of atmospheric transport and the spatial pattern of fluxes (dominated by emissions from localized sources with high population density). In a similar numerical experiment designed to infer $\mathrm{CO}$ fluxes at high spatial resolution based on measurements at Harvard Forest and on a priori fluxes from emission inventories (see G03b), the assumption of a Gaussian a priori uncertainty ( $50 \%$ of the emissions) led to significant negative emissions for localized areas with large a priori emissions, even at larger length scales $(l=100 \mathrm{~km})$. A similar problem has to be expected when including fossil fuel fluxes for $\mathrm{CO}_{2}$ to the state vector. In these cases, a lognormal distribution has to be assumed, and the optimization is not simply a matrix inversion, but has to involve iterative searches, cf. Manning et al. (2003).

The results strongly support that specifying the off diagonal elements of the covariance matrices is crucial for Bayesian flux inversions. An incorrect length scale for the spatial covariance of the a priori guess usually leads to either significant under- or overestimation of the posterior uncertainties. Underestimation of uncertainty is equivalent to overly confident estimates, or biases in the retrievals. Overestimation of uncertainty is equivalent to not using all available information, but instead regarding it as noise; in this case the retrieval will not be biased, but less accurate. The basic underlying principle is that when "defining" the off diagonal elements of prior covariance matrices not in accordance with the factual behavior of the uncertainties, we change the information contained in the prior. As shown in Fig. 11, underestimation of uncertainty occurs in both cases, when using a too large or a too short length scale compared to the actual scale.
It is important to remember that the spatial scales of the a priori uncertainty correlation are closely related to scales of processes that are not included or not properly represented in the flux model. Thus, as stated in the introduction, the ideal way to define the prior uncertainty covariance matrix would be to investigate the spatial (and temporal) correlations of the residuals between the a priori flux model and such measurements that went into the flux model development, or in the case of a state vector consisting of sensitivities to environmental drivers as presented here, to investigate the spatial correlations of these sensitivities for a given class of vegetation. In this regard it is of course helpful if these measurements (flux measurements, process studies) are made in a spatial arrangement that would allow deriving spatial statistics properties (e.g. fitting a variogram). If the datasets do not allow this, one has to adopt other ways to derive the a priori uncertainty covariance matrix. The simplest option is to just assume a certain spatial correlation, as has been done for some global scale inversions cf. Peylin et al. (2001); the assumed correlation has to be tested however with respect to its impact on the retrieval (at least the posterior uncertainty should not be underestimated). A step further is to derive the a priori uncertainty matrix from the spread between different models (Rödenbeck et al., 2003); but one has to be aware of the fact that model to model differences don't necessarily have to resemble residuals between a given model and the truth, given that models often share a similar structure and input data. The assumed correlation could also be based on knowledge of the spatial scales of the processes not represented by the model, e.g. by comparing to a more sophisticated model that includes more detailed processes.

It is also an option to treat such a length scale as an unknown, and to optimize not only for fluxes (or sensitivities to environmental drivers), but also for these length scales. This was for example suggested by Michalak et al. (2004), who used a very simple flux model (the only a priori information was that land and ocean have a different flux), but solved for parameters describing the prior covariance model (a length scale and a variance for land and ocean). However, whichever method is used to derive the a priori uncertainty covariance matrix, it is important to ensure these assumptions are conservative in that claimed uncertainties in derived fluxes (posterior estimates) are not underestimating true uncertainties.

Obviously, there is no simple recipe that would allow defining a length scale $l$, independent of the true length scale $l_{\text {true }}$, such that the posterior uncertainty remains conservative in the sense that the retrieval remains unbiased (estimated posterior uncertainty not smaller than actual the actual uncertainty, see Fig. 11). A common diagnostic to assess whether the correct assumptions about uncertainties went into the optimization is the reduced $\chi^{2}$, i.e. the cost function (weighted sum of squares at the optimum divided by the numbers of degrees of freedom). As expected, reduced $\chi^{2}$ values close to 1 are found for $l=l_{\text {true }}$, (Fig. 12, filled circles). For length 


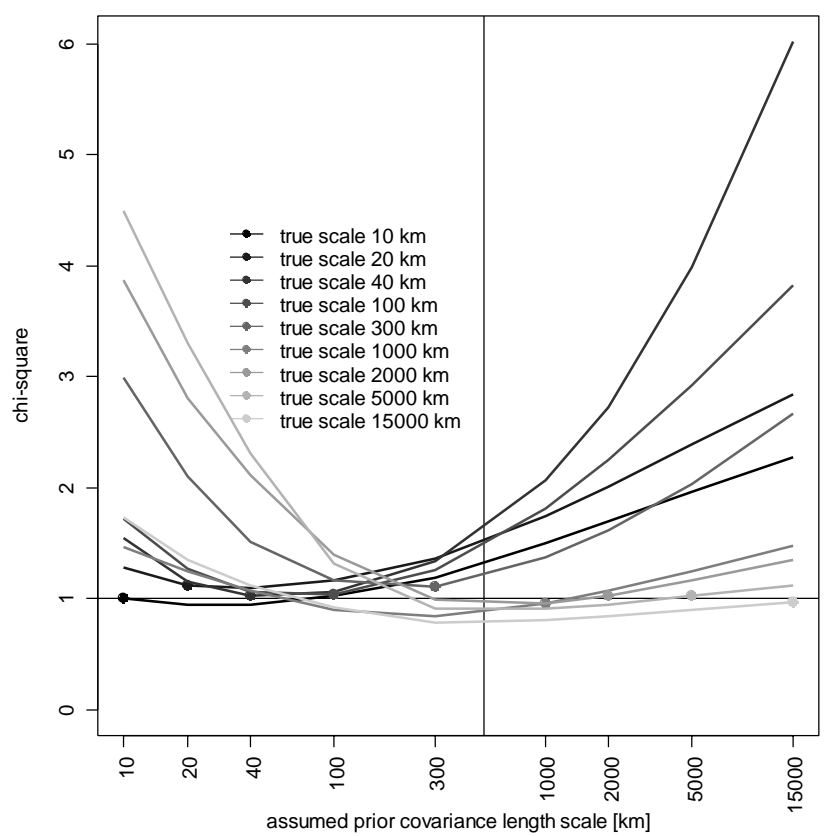

Fig. 12. Reduced $\chi^{2}$ plotted against assumed prior covariance scale l. Grayscales and lines are similar to Fig. 11, with filled circles plotted for $l=l_{\text {true }}$.

scales $l$ that are very different from $l_{\text {true }}$, reduced $\chi^{2}$ values increase up to more than 4 . This seems to indicate that when including the lengthscale $l$ in the state vector, there is a potential for optimizing it in the retrieval. However, values of reduced $\chi^{2}$ around 1 are also found for cases with length scales $l$ that are very different from $l_{\text {true }}$, for example for an assumed scale of $l=40 \mathrm{~km}$ at $l_{\text {true }}=15000 \mathrm{~km}$. Similarly, when plotting the underestimation of uncertainty (i.e. the ratio of actual over assumed posterior uncertainty) against the corresponding reduced $\chi^{2}$ values (Fig. 13), it becomes obvious that a reduced $\chi^{2}$ value of close to unity is a necessary, but not a sufficient sign for a correct posterior uncertainty. Thus the reduced $\chi^{2}$ diagnostics can help to select the appropriate prior uncertainty covariance, but it does not guarantee unbiased retrievals. Therefore, we recommend investigating different possible combinations of true and assumed length scales in order to assure that the final choice is a conservative one.

Next steps will be to use the ROAM framework in this formulation to quantitatively merge top-down constrains from atmospheric observations with bottom-up constraints. Therefore the simple flux model GSB will therefore be replaced by a more sophisticated one such as the VPM (Vegetation Photosynthesis Model, Xiao et al., 2004), which will result in significant improvements of the representation of spatial patterns in biosphere-atmosphere exchange fluxes. Further, dominant transport model uncertainties such as caused by inaccuracies in assimilated winds (Lin and Gerbig, 2005), but also as caused by inappropriate vertical mixing within

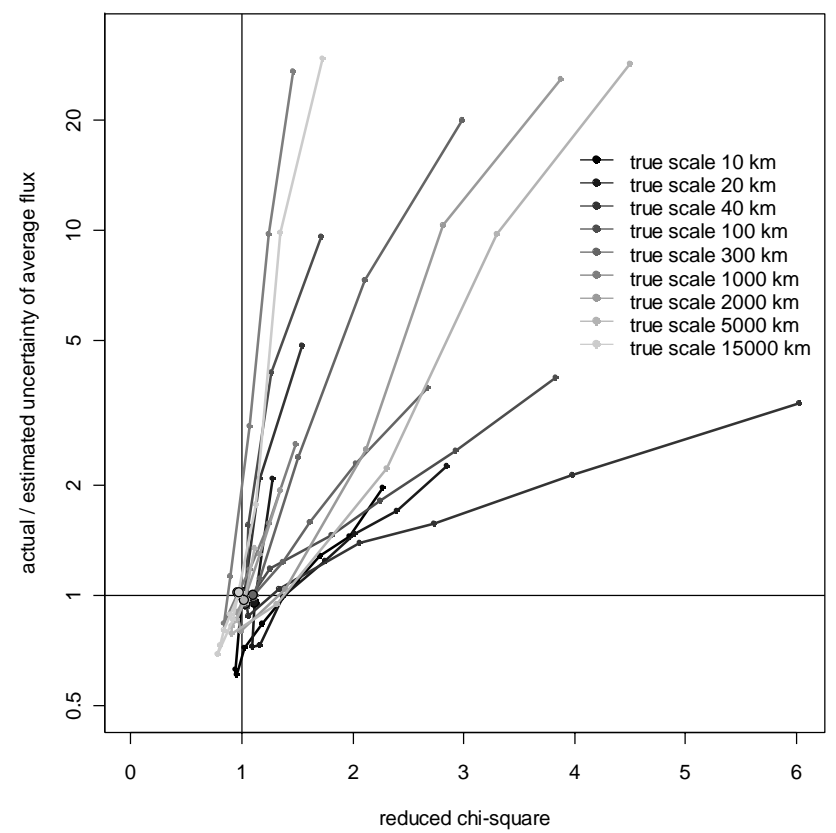

Fig. 13. Ratio of actual to estimated posterior uncertainty of flux average plotted against reduced $\chi^{2}$. Grayscales and lines are similar to Fig. 11, and larger filled circles are plotted for $l=l_{\text {true }}$.

the boundary layer (inaccurate mixed layer height, cf. G03b) need to be (and will be) taken into account via error propagation; this will finally allow for reliable retrievals of regional scale biosphere-atmosphere exchange fluxes.

Acknowledgements. We gratefully acknowledge T. J. Conway from NOAA CMDL for providing remote station and aircraft data from the CMDL network. We also would like to acknowledge C. Roedenbeck for helpful discussions.

Edited by: W. E. Asher

\section{References}

Bakwin, P. S., Tans, P. P., Zhao, C., Ussler, W. I., and Quesnell, E.: Measurements of carbon dioxide on a very tall tower, Tellus, 47B, 535-549, 1995.

Baldocchi, D., Falge, E., Gu, L. H., Olson, R., Hollinger, D., Running, S., Anthoni, P., Bernhofer, C., Davis, K., Evans, R., Fuentes, J., Goldstein, A., Katul, G., Law, B., Lee, X. H., Malhi, Y., Meyers, T., Munger, W., Oechel, W., Paw U, K. T., Pilegaard, K., Schmid, H. P., Valentini, R., Verma, S., Vesala, T., Wilson, K., and Wofsy, S.: FLUXNET: A new tool to study the temporal and spatial variability of ecosystem-scale carbon dioxide, water vapor, and energy flux densities, Bull. Amer. Meteorol. Soc., 82(11), 2415-2434, 2001.

Barford, C. C., Wofsy, S. C., Goulden, M. L., Munger, J. W., Pyle, E. H., Urbanski, S. P., Hutyra, L., Saleska, S. R., Fitzjarrald, D., and Moore, K.: Factors controlling long- and short-term sequestration of atmospheric $\mathrm{CO}_{2}$ in a mid-latitude forest, Science, 294, 1688-1691, 2001. 
Cressie, N. A. C.: Statistics for spatial data, John Wiley and Sons, New York, 900, 1993.

Friedlingstein, P., Dufresne, J.-L., Cox, P. M., and Rayner, P.: How positive is the feedback between climate change and the carbon cycle?, Tellus Series B-Chemical and Physical Meteorology, 55(2), 692-700, 2003.

Gerbig, C., Lin, J. C., Wofsy, S. C., Daube, B. C., Andrews, A. E., Stephens, B. B., Bakwin, P. S., and Grainger, C. A.: Towards constraining regional scale fluxes of $\mathrm{CO} 2$ with atmospheric observations over a continent: 1. Observed Spatial Variability from airborne platforms, J. Geophys. Res., 108(D24), 4756, doi:10.1029/2002JD003018, 2003a.

Gerbig, C., Lin, J. C., Wofsy, S. C., Daube, B. C., Andrews, A. E., Stephens, B. B., Bakwin, P. S., and Grainger, C. A.: Towards constraining regional scale fluxes of $\mathrm{CO} 2$ with atmospheric observations over a continent: 2. Analysis of COBRA data using a receptor-oriented framework, J. Geophys. Res., 108(D24), 4757, doi:10.1029/2003JD003770, 2003b.

GLOBALVIEW-CO ${ }_{2}$ : Cooperative Atmospheric Data Integration Project - Carbon Dioxide, CD-ROM, NOAA CMDL, Boulder, Colorado (also available on Internet via anonymous FTP to ftp. cmdl.noaa.gov, Path: ccg/co2/GLOBALVIEW), 2002.

Gloor, M., Fan, S.-M., Pacala, S., and Sarmiento, J.: Optimal sampling of the atmosphere for purpose of inverse modeling: A model study, Global Biogeochem. Cycles, 14(1), 407-428, 2000.

Gurney, K. R., Law, R. M., Denning, A. S., Rayner, P. J., Baker, D., Bousquet, P., Bruhwiler, L., Chen, Y.-H., Ciais, P., Fan, S., Fung, I. Y., Gloor, M., Heimann, M., Higuchi, K., John, J., Maki, T., Maksyutov, S., Masarie, K., Peylin, P., Prather, M., Pak, B. C., Randerson, J., Sarmiento, J., Taguchi, S., Takahashi, T., and Yuen, C.-W.: Towards robust regional estimates of $\mathrm{CO}_{2}$ sources and sinks using atmospheric transport models, Nature, 415(6872), 626-630, 2002.

Gurney, K. R., Law, R. M., Denning, A. S., Rayner, P. J., Pak, B. C., Baker, D., Bousquet, P., Bruhwiler, L., Chen, Y. H., Ciais, P., Fung, I. Y., Heimann, M., John, J., Maki, T., Maksyutov, S., Peylin, P., Prather, M., and Taguchi, S.: Transcom 3 inversion intercomparison: Model mean results for the estimation of seasonal carbon sources and sinks, Global Biogeochem. Cycles, 18(1), GB1010, doi:10.1029/2003GB002111, 2004.

Intergovernmental Panel on Climate Change (IPCC): Climate Change 2001: The Scientific Basis, Cambridge Univ. Press, New York, 944, 2001.

Kaminski, T., Rayner, P. J., Heimann, M., and Enting, I. G.: On aggregation errors in atmospheric transport inversions, J. Geophys. Res., 106(D5), 4703-4715, 2001.

Lin, J. C. and Gerbig, C.: Accounting for the effect of transport errors on tracer inversions, Geophys. Res. Lett., 32(1), L01802, doi:10.1029/2004GL021127, 2005.

Lin, J. C., Gerbig, C., Wofsy, S. C., Andrews, A. E., Daube, B. C., Davis, K. J., and Grainger, C. A.: A near-field tool for simulating the upstream influence of atmospheric observations: the Stochastic Time-Inverted Lagrangian Transport (STILT) model, J. Geophys. Res., 108(D16), 4493, doi:10.1029/2002JD003161, 2003.
Lin, J. C., Gerbig, C., Wofsy, S. C., Andrews, A. E., Daube, B. C., Grainger, C. A., Stephens, B. B., Bakwin, P. S., and Hollinger, D. Y.: Measuring fluxes of trace gases at regional scales by Lagrangian observations: Application to the $\mathrm{CO}_{2}$ Budget and Rectification Airborne (COBRA) study, J. Geophys. Res.-A., 109, D15304, doi:10.1029/2004JD004754, 2004.

Manning, A. J., Ryall, D. B., Derwent, R. G., Simmonds, P. G., and O'Doherty, S.: Estimating European emissions of ozonedepleting and greenhouse gases using observations and a modeling back-attribution technique, J. Geophys. Res.-A., 108(D14), 4405, doi:10.1029/2002JD002312, 2003.

Michalak, A. M., Bruhwiler, L., and Tans, P. P.: A geostatistical approach to surface flux estimation of atmospheric trace gases, J. Geophys. Res.-A., 109(D14), D14109, doi:10.1029/2003JD004422, 2004.

Peylin, P., Bousquet, P., and Ciais, P.: Response to: Inverse modeling of atmospheric carbon dioxide fluxes, Science, 294, 259-260, 2001

Peylin, P., Rayner, P. J., Bousquet, P., Carouge, C., Hourdin, F., Heinrich, P., Ciais, P., and contributors, A.: Daily $\mathrm{CO}_{2}$ flux estimates over Europe from continuous atmospheric measurements: 1, inverse methodology, Atmos. Chem. Phys., 5, 3173-3186, 2005 ,

SRef-ID: 1680-7324/acp/2005-5-3173.

Rödenbeck, C., Houweling, S., Gloor, M., and Heimann, M.: CO2 flux history 1982-2001 inferred from atmospheric data using a global inversion of atmospheric transport, Atmos. Chem. Phys., 3, 1919-1964, 2003,

SRef-ID: 1680-7324/acp/2003-3-1919.

Rodgers, C. D.: Inverse methods for atmospheric sounding: theory and practice, World Scientific, Singapore, 238, 2000.

Rogers, E., Deaven, D. G., and Dimego, G. J.: The regional analysis system for the operational early Eta-model: original $80-\mathrm{km}$ configuration and recent changes, Weat. Forecasting, 10(4), 810 $825,1995$.

Schimel, D. S., House, J. I., Hibbard, K. A., Bousquet, P., Ciais, P., Peylin, P., Braswell, B. H., Apps, M. J., Baker, D., Bondeau, A., Canadell, J., Churkina, G., Cramer, W., Denning, A. S., Field, C. B., Friedlingstein, P., Goodale, C., Heimann, M., Houghton, R. A., Melillo, J. M., Moore III, B., Murdiyarso, D., Noble, I., Pacala, S. W., Prentice, I. C., Raupach, M. R., Rayner, P. J., Scholes, R. J., Steffen, W. L., and Wirth, C.: Recent patterns and mechanisms of carbon exchange by terrestrial ecosystems, Nature, 414, 169-172, 2001.

Wang, Y. P.: Estimating regional terrestrial carbon fluxes for the Australian continent using a multiple-constraint approach: II, The atmospheric constraint, Tellus, 55B, 290-304, 2003.

Wofsy, S. C., Goulden, M. L., Munger, J. W., Fan, S.-M., Bakwin, P. S., Daube, B. C., Bassow, S. L., and Bazzaz, F. A.: Net exchange of $\mathrm{CO}_{2}$ in a mid-latitude forest, Science, 260, 1314-1317, 1993.

Wofsy, S. C. and Harriss, R. C.: The North American Carbon Program (NACP), NACP Committee of the U.S. Interagency Carbon Cycle Science Program, U.S. Global Change Research Program, Washington D.C., 2002.

Xiao, X. M., Hollinger, D., Aber, J., Goltz, M., Davidson, E. A., Zhang, Q. Y., and Moore III, B.: Satellite-based modeling of gross primary production in an evergreen needleleaf forest, Rem. Sens. Environ., 89(4), 519-534, 2004 